\title{
Fistola arterovenosa carotido-giugulare: una complicazione del catetere temporaneo per emodialisi
}

\author{
Da: American Journal of Nephrology 1995; 15: 332-6 \\ El-Shahawy MA, Khilani $H$
}

$\mathrm{U}$ n uomo di 44 anni viene ricoverato in ospedale con intossicazione da anfetamine, rabdomiolisi ed insufficienza renale acuta oligurica. All'ingresso si presenta febbrile, tachicardico, tachipnoico, con pressione arteriosa normale, letargico. Il dipstick delle urine rileva sangue e proteinuria $1+$, ma il sedimento urinario è negativo; le prove ematochimiche dimostrano severa iperkaliemia $(6.8 \mathrm{mEq} / \mathrm{l})$, ipercreatininemia $(5.8 \mathrm{mg} / \mathrm{dl})$, elevato BUN $(54 \mathrm{mg} / \mathrm{dl})$, iperuricemia $(24.4 \mathrm{mg} / \mathrm{dl})$, valori estremamente levati di CPK (190.000 U/1) e LDH (5.336 U/1); l'ecografia renale è nella norma. Viene posizionato un catetere a doppio lume per emodialisi nella vena succlavia $d x$ e viene iniziata emodialisi extracorporea; dopo due settimane il flusso ematico attraverso tale catetere risulta scarso ed il catetere in vena succlavia viene sostituito con un nuovo catetere in vena giugulare $\mathrm{dx}$; dopo tale posizionamento il paziente dimostra gonfiore al collo o viene trasferito ad un ospedale più attrezzato.

In tale sede viene riscontrato un rigonfiamento alla metà $\mathrm{dx}$ del collo con dolorabilità alla palpazione, fremito all'ascoltazione alla metà $\mathrm{dx}$ del collo, deviazione della trachea a sn. Un $\mathrm{Rx}$ dimostra che il catetere venoso centrale è correttamente posizionato. Un esame doppler dei vasi del collo rileva una fistola artero-venosa tra la vena giugulare $\mathrm{dx}$ e la arteria carotide comune dx; un'angiografia carotidea conferma la fistola a livello della giunzione tra sesta e settima vertebra cervicale. Viene effettuata una esplorazione chirurgica che rileva soffusione emorragica del lato destro del collo, ematoma di $3 \mathrm{~cm}$ tra arteria carotide comune e vena giugulare interna $\mathrm{dx}$, un difetto di $3 \mathrm{~mm}$ nella parete mediale della vena giugulare interna $\mathrm{dx}$ e un corrispondente difetto di $3 \mathrm{~mm}$ nella parete laterale dell'arteria carotide comune $\mathrm{dx}$; tali difetti vengono riparati.
Le sedute emodialitiche vengono proseguite dopo posizionamento di un catetere in vena femorale $\mathrm{dx}$. Dopo una settimana il paziente guarisce dalla insufficienza renale acuta e l'emodialisi viene interrotta.

L'interesse di questo caso clinico è dato in primo luogo dalla inedita segnalazione di fistola artero-venosa tra arteria carotide e vena giugulare in seguito a posizionamento di catetere venoso in giugulare per emodialisi: si tratta di una complicanza traumatica importante, che richiede una rapida diagnosi e la pronta effettuazione di un delicato intervento di chirurgia vascolare.

I tipi di catetere venoso più usati in dialisi sono i femorali, i succlavi ed i giugulari. La cateterizzazione della vena femorale è ormai poco usata a causa di interferenze con la deambulazione, sanguinamento, infezione, trombosi venosa profonda. Anche il cateterismo della vena succlavia può dare adito a numerose complicanze a breve termine: traumatismo di strutture centrali, infezioni, trombosi venosa, puntura dell'arteria succlavia, lacerazione venosa, ematoma, emotorace, pneumotorace, perforazione cardiaca, tamponamento pericardico. Il cateterismo della vena succlavia è associato inoltre ad una elevata incidenza di complicanze a lungo termine, rappresentate soprattutto dalla stenosi della vena succlavia.

Negli ultimi anni è stato proposto il cateterismo della vena giugulare, che dovrebbe garantire una maggiore sicurezza in base alla rarità delle complicanze a lungo termine. Gli Autori sottolineano comunque che anche il catetere giugulare è associato ad un elevato rischio di complicanze a breve termine, di cui il caso clinico in oggetto rappresenta un esempio inedito.

In definitiva, l'uso di qualunque catetere venoso per emodialisi deve essere associato ad una attenta sorveglianza del paziente, soprattutto nei primi giorni successivi al posizionamento. 